\title{
Selectagerm: A new approach to clinical bacteriology
}

\author{
P. W. GREAVES
}

From the Public Health Laboratory, City and Sherwood Hospitals, Hucknall Road, Nottingham

SYNOPSIS A method of processing clinical specimens received at the laboratory on cotton wool swabs is described. The method uses a range of selective culture media to assist in the isolation of potential pathogens from mixed cultures. Because the culture media are distributed in 2 millilitre volumes in the small squares of divided plastic petri dishes it is economically possible to use the same series for every specimen. This facilitates the rapid processing of large numbers of swabs and allows the isolation of pathogens from sites where they might otherwise go unnoticed.

Many laboratories use selective culture media as well as non-inhibitory media when seeking specific pathogens from sites with a normal bacterial flora or when a mixed infection is suspected. Material from the initial inoculum on a non-selective plate must be serially spread over a large area of medium so that well isolated individual colonies of all of the bacteria present may be detected. However, with a suitable selective medium, meticulous 'plating out' of the inoculum is usually unnecessary; only a small portion of the surface need be used and several different specimens may be examined on one plate.

This paper describes a method of using 10 selective culture media which among them will isolate all of the aerobic bacteria commonly encountered in clinical bacteriology. The media are distributed in $2 \mathrm{ml}$ volumes in each of the 25 squares of 4 in. divided petri dishes (Dyos Plastics Ltd.) and inoculated with a standard loopful $(0.01 \mathrm{ml})$ of a suspension of bacteria derived from clinical specimens received at the laboratory on cotton wool swabs. Because of the small volume of selective medium involved the same basic set can be economically employed for a wide variety of specimens. This makes possible the isolation of certain pathogens from sites where they might otherwise go unnoticed.

\section{Materials and Methods}

The following culture media were employed.

NO. 1 FOR Staphylococcus aureus

Oxoid Dnase agar (CM 321) with $0.025 \%$ methyl green (CI 42585), polymyxin ( 500 units $/ \mathrm{ml}$ ), nalidixic acid $(10 \mu \mathrm{g} / \mathrm{ml})$, and amphotericin B $(2 \cdot 5 \mu \mathrm{g} / \mathrm{ml})$.

Received for publication 19 January 1973.
NO. 2 FOR STREPTOCOCCI AND PNEUMOCOCCI Oxoid BAB no. 2 (CM 271) with 7\% defibrinated horse blood, polymyxin (50 units/ml), nalidixic acid $(10 \mu \mathrm{g} / \mathrm{ml})$, and $1: 500000$ crystal violet.

NO. 3 FOR PATHOGENIC Neisseriae

Oxoid DST agar (CM 261) with 7\% lysed horse blood, polymyxin (15 units $/ \mathrm{ml})$, vancomycin $(5$ $\mu \mathrm{g} / \mathrm{ml})$, trimethoprim $(5 \mu \mathrm{g} / \mathrm{ml})$, and amphotericin B $(2 \cdot 5 \mu \mathrm{g} / \mathrm{ml})$.

NO. 4 FOR CORYNEBACTERIA

Oxoid Hoyle's base (CM 83) with $7 \%$ lysed horse blood, $\mathbf{0 . 0 3 5 \%}$ potassium tellurite, and amphotericin B $(2 \cdot 5 \mu \mathrm{g} / \mathrm{ml})$.

\section{NO. 5 FOR Haemophilus influenzae}

Chocolate agar containing Oxoid BAB no. 2 (CM 271), $7 \%$ defibrinated horse blood, bacitracin $(1.5$ units $/ \mathrm{ml})$, and amphotericin B $(2.5 \mu \mathrm{g} / \mathrm{ml})$.

NO. 6 FOR Candida albicans

Oxoid malt extract agar (CM 59) with 0.014\% potassium tellurite and chloramphenicol $(40 \mu \mathrm{g} / \mathrm{ml})$.

NO. 7 FOR Pseudomonas aeruginosa

Oxoid BAB no. 2 with $0.025 \%$ cetrimide and nalidixic acid $(10 \mu \mathrm{g} / \mathrm{ml})$.

NO. 8 FOR THE Proteus GROUP

Phenylalanine agar (Shaw and Clarke, 1955) with polymyxin ( 500 units $/ \mathrm{ml})$, bacitracin $(5$ units $/ \mathrm{ml})$, and amphotericin B $(2 \cdot 5 \mu \mathrm{g} / \mathrm{ml})$.

NO. 9 FOR Klebsiella aerogenes

Oxoid Christensen's urea agar (CM 53) with carbenicillin $(75 \mu \mathrm{g} / \mathrm{ml})$ and amphotericin B $(2.5 \mu \mathrm{g} / \mathrm{ml})$. 
NO. 10 FOR Esch. coli AND OTHER COLIFORM ORGANISMS

Oxoid MacConkey agar (CM 7) with $0.5 \%$ sucrose, bacitracin ( 5 units $/ \mathrm{ml})$, and amphotericin B $(2 \cdot 5$ $\mu \mathrm{g} / \mathrm{ml})$.

Each of these culture media was tested for the following properties.

For its ability to support the growth of different strains of the appropriate test organism from a small inoculum. Except for three strains of $C$. diphtheriae from the National Collection of Type Cultures each of the organisms used was freshly isolated from routine clinical material on a non-inhibitory medium. As far as possible only epidemiologically unrelated strains were employed. Viable counts were determined on blood agar and on a selective medium by the method of Miles and Misra (1938).

For its ability to inhibit different numbers of 'contaminating' organisms in a series of artificially prepared mixtures. Twenty-five mixtures, each containing Staphylococcus aureus, Streptococcus pyogenes, Neisseria gonorrhoeae, Haemophilus influenzae, Corynebacterium diphtheriae, Candida albicans, Pseudomonas aeruginosa, Proteus mirabilis, Klebsiella aerogenes, and Escherichia coli, but in different proportions, were inoculated on batches of media which had been stored at $4^{\circ} \mathrm{C}$ for varying periods up to 10 days.

For its performance in routine clinical bacteriology in comparison with the methods currently employed in this laboratory and in a second independent laboratory. Material received on cotton wool swabs was processed by first inoculating the plates of culture media normally employed and then breaking the swab into a bijou bottle containing $1 \mathrm{ml}$ nutrient broth. After standing at room temperature for five to 10 minutes to allow the swabs to become thoroughly moistened the bijoux bottles were shaken vigorously in the horizontal plane and a standard loopful of the resulting suspension was inoculated onto individual squares of each selective culture medium.

\section{Results}

MEDIUM NO. 1 FOR Staphylococcus aureus This medium was designed to isolate Staphylococcus aureus and to inhibit almost all other organisms, including Staphylococcus epidermidis. It is a combination of the selective medium of Finegold and Sweeney (1961) and the modification by Smith, Hancock, and Rhoden (1969) of the Dnase medium described by Jeffries, Holtman, and Guse (1957).

Most coagulase-positive staphylococci grew well and showed a positive Dnase reaction after over2 night incubation, but about $10 \%$ grew poorly and isolated colonies of these were Dnase negative. These could be rapidly differentiated from the few strains of coagulase-negative staphylococci which grew on this medium by placing a large inoculum on a thin plate of conventional Dnase agar and testing for Dnase production after incubation at $37^{\circ} \mathrm{C}$ for four hours (Jeffries et al, 1957).

Most strains of streptococci grew on the medium, and some were Dnase positive. No other organisms tested grew on this medium and there was no significant difference in the recovery of the test organisms from artificial mixtures after the plates had been stored at $4^{\circ} \mathrm{C}$ for 10 days.

\section{MEDIUM NO. 2 FOR STREPTOCOCCI}

This is crystal violet blood agar (Garrod, 1942) incorporating nalidixic acid in order to inhibit 'coliform' organisms (Beerens and Tahon-Castel, 1966) and polymyxin to inhibit Pseudomonas aeruginosa (Kidson, 1967).

All of the strains of streptococci examined grew equally well on the selective medium and on blood agar, giving typical haemolytic reactions. None of the other bacterial species tested were capable of growth èven when present in large numbers and there was no deterioration in performance after storage at $4^{\circ} \mathrm{C}$ for 10 days.

MEDIUM NO. 3 FOR PATHOGENIC NEISSERIAE This medium (Lancet, 1970; Phillips, Humphrey, Middleton, and Nicol, 1972) has been used in this laboratory for the isolation of Neisseria gonorrhoeae for the past two years.

Most strains of gonococci and all of the strains of meningococci used in this study grew well and none of the other bacteria tested grew on the medium even when present in large numbers. However, three strains of gonococci failed to grow and problems may arise if such strains are locally numerous. There was no difference in performance of the medium after storage at $4^{\circ} \mathrm{C}$ for 10 days.

MEDIUM NO. 4 FOR CORYNEBACTERIA

This is the traditional Hoyle's medium (Hoyle, 1941) which has been used for many years for the isolation of Corynebacterium diphtheriae. Some strains of staphylococci and streptococci can grow on this medium when present in large numbers, but the colonial forms are easily distinguished from those of the corynebacteria. Each of the stock strains of the diphtheria bacillus grew well and viable counts were equivalent to those on blood agar. The medium can be stored for more than 10 days at $4^{\circ} \mathrm{C}$ without deterioriation. 
MEDIUM NO. 5 FOR Haemophilus influenzae

This is described by Jarvis and Ewins (1968) and during the preliminary trials was the least satisfactory of the media since it failed to inhibit enterobacteria, hence isolation of Haemophilus influenzae from the artificial mixtures was difficult although there was seldom any trouble in isolating this organism from clinical material.

The inhibitory effect of the bacitracin was reduced on prolonged storage and some strains of staphylococci were then able to grow.

\section{MEDIUM NO. 6 FOR Candida albicans}

This has been in regular use in this laboratory for many years and is highly selective for organisms of the Candida group. Colonies are small after overnight incubation and there is seldom any evidence of tellurite reduction at this stage; best results are obtained after incubation for $\mathbf{4 8}$ hours. This medium also stores well.

\section{MEDIUM NO. 7 FOR Pseudomonas aeruginosa}

This was described by Lilly and Lowbury (1972). All of the strains of Pseudomonas aeruginosa examined grew well and gave a positive oxidase reaction after overnight incubation. The results of the viable counts were equivalent to those on blood agar and most strains showed classical pigmentation, although in some cases this was more easily seen after 48 hours. None of the other species of bacteria examined grew on this medium and there was no significant difference in performance after storage at $4^{\circ} \mathrm{C}$ for 10 days.

Medium No. 8 for the Proteus group This medium was devised for the immediate group identification of Proteus strains. All of the strains of Proteus examined grew freely on the medium and gave a positive reaction for phenylalanine deaminase after overnight incubation. As well as the usual test strains, several hundred strains of other 'coliform' bacilli isolated from the urinary tract were examined. None grew on the freshly prepared medium, but the plates were less inhibitory after storage at $4^{\circ} \mathrm{C}$ for more than seven days. No strains of Serratia marcescens were available for testing; these would be expected to grow on the medium but would give a negative reaction in the test for phenylalanine deaminase.

MEDIUM NO. 9 FOR Klebsiella aerogenes This was designed for the immediate recognition of Klebsiella aerogenes and its differentiation from mucoid strains of Escherichia coli. The majority of strains of Klebsiellae grew well on the medium and proved capable of hydrolysing urea, but a few strains proved sensitive to the concentration of carbenicillin employed. Occasional species of Enterobacter and strains of carbenicillin-resistant Pseudomonas aeruginosa also grew but did not hydrolyse urea. There was no significant difference in performance of the medium after storage at $4^{\circ} \mathrm{C}$ for 10 days.

MEDIUM NO. 10 FOR Escherichia coli AND OTHER COLIFORM ORGANISMS

Pseudomonas aeruginosa, Klebsiella aerogenes, and all species of Proteus grow on this medium, as well as Escherichia coli and other coliform organisms. It is thus difficult to separate Escherichia coli from mixtures of enterobacterial species. The medium is included in order to detect 'coliforms' other than those for which special culture media have been devised. The majority of Gram-positive organisms are inhibited for at least $\mathbf{1 0}$ days after preparation.

The results of examination of the artificial mixtures showed that any organism except Haemophilus influenzae and Escherichia coli could be isolated on the appropriate selective medium if it was present in numbers greater than 500 cells per $\mathrm{cm}^{3}$, irrespective of the numbers of other organisms present. Small numbers of Escherichia coli and Haemophilus influenzae escaped recognition in the presence of greater numbers of other coliform bacilli. Table I shows the spectrum of growth of the test organisms on these media.

ISOLATIONS FROM CLINICAL SPECIMENS

At this stage, the Selectagerm system seemed sufficiently promising to warrant direct comparison with the methods used in the routine diagnostic laboratory. All swabs received over a period of five days were therefore examined by both methods.

Respiratory specimens, including ear, nose, and throat swabs were examined routinely on blood agar medium and crystal-violet-nalidixic acid blood agar (CVNA) for haemolytic streptococci. Hoyle's medium, Candida medium, and chocolate agar were included where relevant. There were 61 of these.

Vaginal swabs were examined routinely on cysteine-lactose-electrolyte-deficient agar (CLED) and Candida medium. Reduced neomycin blood agar for anaerobic culture and chocolate and crystalviolet-nalidixic acid blood agar media were included where appropriate. There were 91 of these.

Other swabs, including swabs from postoperative wounds and a wide variety of other septic conditions, were examined on blood agar or electrolyte-deficient agar medium according to the site of the infection. Cultures for anaerobes and other specific organisms such as haemolytic streptococci were included where appropriate. There were 85 of these. 


\begin{tabular}{|c|c|c|c|c|c|c|c|c|c|c|}
\hline & \multicolumn{10}{|c|}{ Medium Number } \\
\hline & $l$ & 2 & 3 & 4 & 5 & 6 & 7 & 8 & 9 & 10 \\
\hline Staph aureus & $++t^{1}$ & - & - & $d^{2}$ & - & - & - & - & - & - \\
\hline ", epidermidis & d & - & - & - & - & - & - & - & - & - \\
\hline Strep. pyogenes & $+t+$ & $+t+$ & - & - & - & - & - & - & - & - \\
\hline " agalactiae & $+t+$ & $++t$ & - & - & - & - & - & - & - & - \\
\hline " faecalis & $+t+$ & $+t+$ & - & d & - & - & - & - & - & - \\
\hline " viridans & $+t+$ & $+t+$ & - & d & - & - & - & - & - & - \\
\hline "pneumoniae & + & +++ & - & - & - & - & - & - & - & - \\
\hline$H$. influenzae & - & - & - & - & $++t$ & - & - & - & - & - \\
\hline$P$. septica & - & - & - & - & - & - & - & - & - & - \\
\hline N. gonorrhoeae & - & - & $+t+$ & - & - & - & - & - & - & - \\
\hline N. meningitidis & - & - & +++ & - & - & - & - & - & - & - \\
\hline N. catarrhalis & - & - & - & - & - & - & - & - & - & - \\
\hline C. diphtheriae & - & - & - & $++t$ & - & - & - & - & - & - \\
\hline Ps. aeruginosa & - & - & - & - & $++t$ & - & $+t+$ & - & d & $+t+$ \\
\hline Ac. anitratus & - & - & - & - & $+t+$ & - & - & - & - & $+t+$ \\
\hline Pr. mirabilis & - & - & - & - & $++t$ & - & - & $+t+$ & - & $t+t$ \\
\hline Ct. freundii & - & - & - & - & $+t+$ & - & - & - & - & $+t+$ \\
\hline K. aerogenes & - & - & - & - & $+t+$ & - & - & - & $+t+$ & $+t+$ \\
\hline E. coli & - & - & - & - & $+t+$ & - & - & - & - & $++t$ \\
\hline Sh. sonnei & - & - & - & - & $+t+$ & - & - & - & - & $+t+$ \\
\hline Ent. cloacae & - & - & - & - & $+t+$ & - & - & - & ++ & $+t+$ \\
\hline Candida albicans & - & - & - & - & - & $+t+$ & - & - & - & - \\
\hline
\end{tabular}

Table I List of organisms examined and spectrum of growth on the various selective media

$1++=$ growth equivalent to that on blood agar.

$2 \mathrm{~d}=$ doubtful, some strains are capable of growth.

Fifty-six of the 237 swabs showed no bacterial growth with either method. In a further 76 specimens the same organisms were isolated by both and when two or more organisms were isolated from the same specimen the relative proportions of each were similar. From the remaining 105 specimens the service laboratory isolated 44 strains not detected by Selectagerm and 88 strains were isolated by Selectagerm alone. With the exception of Staphylococcus epidermidis, which Selectagerm is designed to inhibit, none of the strains isolated by the service laboratory alone produced more than 50 colonies, and several less than 10 colonies, on any plate, whereas many of the strains only isolated by Selectagerm were present in profuse or confluent growth on the appropriate selective medium. The results of this trial are shown in table II.

Since this trial compared the results of the Selectagerm system with those from a laboratory where there was already a wide use of selective culture media, a further comparative trial was undertaken with material that had already been examined in another, independent laboratory. The results, which closely resembled those from the earlier exercise, are shown in table III.

\section{Discussion}

The Selectagerm system offers three main advantages over more conventional bacteriological methods.

\begin{tabular}{|c|c|c|c|c|c|}
\hline & \multicolumn{3}{|c|}{ Number of Isolates } & \multicolumn{2}{|l|}{ Proportion of Isolates } \\
\hline & Both Methods & $\begin{array}{l}\text { Service Laboratory } \\
\text { Only }\end{array}$ & Selectagerm Only & $\begin{array}{l}\text { Service Laboratory } \\
(\%)\end{array}$ & Selectagerm $(\%)$ \\
\hline $\begin{array}{l}\text { Staph. epidermidis } \\
\text { Staph. aureus } \\
\beta \text {-streptococci } \\
\text { Enterococci } \\
\text { Other streptococci } \\
\text { H. influenzae } \\
\text { Esch. coli } \\
\text { Klebsiella } \\
\text { Proteus } \\
\text { Ps. aeruginosa } \\
\text { Candida }\end{array}$ & $\begin{array}{r}6 \\
22 \\
7 \\
30 \\
59 \\
2 \\
30 \\
2 \\
8 \\
7 \\
11\end{array}$ & $\begin{array}{r}19 \\
8 \\
5 \\
4 \\
1 \\
0 \\
4 \\
0 \\
0 \\
0 \\
3\end{array}$ & $\begin{array}{r}5 \\
11 \\
4 \\
12 \\
9 \\
22 \\
12 \\
3 \\
6 \\
2 \\
2\end{array}$ & $\begin{array}{r}83 \\
73 \\
75 \\
74 \\
87 \\
8 \\
8 \\
74 \\
40 \\
78 \\
78 \\
88\end{array}$ & $\begin{array}{r}37 \\
81 \\
69 \\
91 \\
99 \\
100 \\
91 \\
100 \\
100 \\
100 \\
81\end{array}$ \\
\hline Total & 184 & 44 & 88 & 72 & 86 \\
\hline Total excluding Staph. epidermidis & 178 & 25 & 83 & 71 & 91 \\
\hline
\end{tabular}

Table II Numbers of bacterial isolates from 237 swabs examined by the Selectagerm method and by conventional laboratory techniques 


\begin{tabular}{|c|c|c|c|c|c|}
\hline & \multicolumn{3}{|c|}{ Number of Isolates } & \multicolumn{2}{|c|}{ Proportion of Isolates } \\
\hline & Both Methods & $\begin{array}{l}\text { Independent } \\
\text { Laboratory }\end{array}$ & Selectagerm & $\begin{array}{l}\text { Independent } \\
\text { Laboratory }(\%)\end{array}$ & Selectagerm $(\%)$ \\
\hline Staph. epidermidis & - & 19 & 4 & 83 & 17 \\
\hline Staph. aureus & 42 & 3 & 10 & 82 & 95 \\
\hline$\beta$-Haemolytic streptococci & 6 & 3 & 1 & 90 & 70 \\
\hline Enterococci & 16 & 2 & 7 & 72 & 92 \\
\hline Other streptococci & 4 & 1 & 2 & 71 & 86 \\
\hline H. influenzae & 2 & - & 1 & 67 & 100 \\
\hline Esch. coli & 15 & 2 & 2 & 89 & 89 \\
\hline Klebsiella & 4 & - & - & 100 & 100 \\
\hline Proteus & 6 & 4 & 1 & 91 & 64 \\
\hline Ps. aeruginosa & 4 & 1 & - & 100 & 80 \\
\hline Candida & 3 & - & - & 100 & 100 \\
\hline Total & 102 & 35 & 28 & 83 & 79 \\
\hline Total excluding Staph. epidermidis & 102 & 16 & 24 & 83 & 89 \\
\hline
\end{tabular}

Table III Numbers of bacterial isolates from 135 swabs examined by the Selectagerm technique and in an independent hospital laboratory

Specimens may be examined on a wide variety of selective culture media at a cost of little more than one additional blood agar plate and organisms may be isolated which would otherwise be missed. Many of the strains isolated can be immediately identified, at least to generic level, by virtue of their growth or specific biochemical reactions on the appropriate culture medium, and the relative proportions of the different organisms present in a lesion can be readily determined.

A surprising feature of the trial was the number of occasions when single organisms were isolated in profuse growth by Selectagerm from specimens which yielded little on no growth by conventional methods. An analysis of the results suggests that this is less likely to happen when swabs travel to the laboratory in transport medium, and that agitation in broth effects a better recovery of organisms from dry swabs than pressure on the surface of agar plates. However, since there are bacteria which will not grow on any of the selective media, and some specimens which yield less than 500 organisms per $\mathrm{cm}^{3}$ of broth suspension, it is still necessary to use a non-inhibitory medium as well as the Selectagerm set.

This system is currently in use at our own laboratory together with reduced neomycin blood agar plates for anaerobic culture.

I should like to thank the staff at Nottingham
Public Health Laboratory for their wholehearted support during the performance of this work, and in particular Dr Malcolm Lewis for his help in the preparation of this paper.

\section{References}

Beerens, H., and Tahon-Castel, M. M. (1966). Milieu à l'acide nalidixique pour l'isolement des streptocoques, $D$. pneumoniae listeria et erisipelothrix. Ann. Inst. Pasteur, 111, 90-93.

Finegold, S. M., and Sweeney, E. E. (1961). New selective and differential medium for coagulase-positive staphylococci allowing rapid growth and strain differentiation. J. Bact., 81, 636-641.

Garrod, L. P. (1942). The selective bacteristatic action of gentian violet. Brit. med. J., 1, 290-291.

Hoyle, L. (1941). A tellurite blood-agar medium for the rapid diagnosis of diphtheria. Lancet, 1, 175-176.

Jarvis, J. D., and Ewins, S. P. (1968). Bacitracin as a selective agent for Haemophilus species. J. med. Lab. Technol., 25, 261-262.

Jeffries, C. D., Holtman, D. F., and Guse, D. G. (1957). Rapid method for determining the activity of microorganisms on nucleic acids. J. Bact., 73, 590-591.

Kidson, A. (1967). A new selective medium for Streptococcus pyogene and other streptococci. J. med. Lab. Technol., 24, 179-186.

Lancet (1970). Leading article. Lancet, 1, pp. 280-281.

Lilly, H. A., and Lowbury, E. J. L. (1972). Cetrimide-nalidixic acid agar as a selective medium for Pseudomonas aeruginosa. $J$. med. Microbiol., 5, 151-153.

Miles, A. A., and Misra, S. S. (1938). The estimation of the bactericidal power of the blood. J. Hyg. (Lond.), 38, 732-748.

Phillips, I., Humphrey, D., Middleton, A., and Nicol, C. S. (1972). Diagnosis of gonorrhoea by culture on a selective medium containing vancomycin, colistin, nystatin and trimethoprim (VCNT): a comparison with Gram staining and immuno-

fluorescence. Brit. J. vener. Dis., 48, 287-292.
Shaw, C., and Clarke, P. H. (1955). Biochemical classification of Proteus and Providence cultures. J. gen. Microbiol., 13, 155-161. $\omega$

Smith, P. B., Hancock, G. A., and Rhoden, D. L. (1969). Improved medium for detecting Deoxyribonuclease producing bacteria. Appl. Microbiol., 18, 991-993. 